\title{
Research Article \\ Optimal Satellite LEO Constellation Design Based on Global Coverage in One Revisit Time
}

\author{
Peng Zong and Saeid Kohani $\mathbb{D}$ \\ Nanjing University of Aeronautics and Astronautics, Astronautics College, Nanjing, Jiangsu 210016, China \\ Correspondence should be addressed to Saeid Kohani; saeid.kohani@nuaa.edu.cn
}

Received 5 August 2019; Accepted 8 November 2019; Published 6 December 2019

Academic Editor: Hikmat Asadov

Copyright ( 2019 Peng Zong and Saeid Kohani. This is an open access article distributed under the Creative Commons Attribution License, which permits unrestricted use, distribution, and reproduction in any medium, provided the original work is properly cited.

\begin{abstract}
This paper presents a globe coverage of constellation satellite in one revisit and the regional coverage at defined latitude. This constellation seems to be very close to optimal under the maximum revisit time comparing to the results in other papers. They are produced easily by using tables and data achievements. These satellites are fully connected by crosslinks sweeping the Earth. This model is more optimal comparing to the conventional constellations that distribute satellites evenly in the space. Since satellites on the LEO orbit are side by side, in most cases, they can maintain communication continuously. This special feature allows all satellites in the constellation connecting ground at any time when a satellite is available to the stations. Length of the crosslink is allowed to reject location connection or real-time data transmission. For example, six satellites in the constellation cover the whole Earth within one revisit time and all the data are collected by two Earth stations for keeping continuous coverage. Thus, the adjacent satellites may be more efficient and provide more coverage.
\end{abstract}

\section{Introduction}

Studies have shown that Walker constellations with given $(T / P / F)$ parameters are optimal for global comprehensive coverage. Under condition that there are not enough satellites for continuous coverage, the max revisit time (MRT) is optimized to minimize number of satellites [4]. However, when the revisit time approaches the orbital period, Walker's solutions are not as effective as genetic algorithms (GA) [1-3, 5]. Normally, the maximum and average revisit time (MRT and ART) can be determined by GA data from the standard curve or called "Pareto Front"; it allows the user to choose the best solution between MRT and ART. The GA method requires a lot of computing resources and time for each case and generally does not provide much information on how to obtain a solution for other species. An important exception is that GA method solutions typically provide sensor switches for false adjacent unplugged interfaces and without gap between them. So, for the purpose of designing a favorable constellation of LEO, for the complete coverage of the Earth, navigation, etc., methods are used, including the genetic algorithm. Genetic algorithm (GA) is an optimization method based on Darwin's natural and genetic selection. These algorithms are different from the traditional optimization techniques because they work with parser encoding instead of their parameters. This encoding is usually performed as a binary string, as was done in this study. The genetic algorithm optimizes and speeds up computation and accuracy of problem solving. This method has been used in several researches by Matthew L. Marcus, Daniele Mortari, William J. Mason, etc., which optimize the design of the orbit. Further details of these researches can be found in References [2, 7] and [6]. The present study takes the idea of using terrestrial footprints of satellites on the ground in the form of bands that are working harmoniously in a revisit time as "Painting the Earth". With "painting ground" standard, we can prevent the disagreement between MRT and ART. The time required to complete full colorization of the Earth is closer to the MRT. There are several ways to arrange satellites and keep their band vicinity. The following are the two methods considered in this study. First, with the COF method (there are two groups of satellites in 8 planes; "there are two groups of four planes," which are configured in a constellation that move obliquely and cover the Earth; the advantage of this 


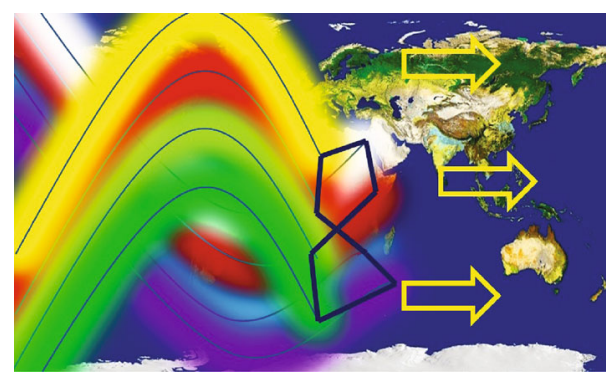

FIgURE 1: Brush lines of 9 satellites in COF $(h=650 \mathrm{~km}, i=60 \mathrm{deg}$, $\varepsilon>10 \mathrm{deg})$.

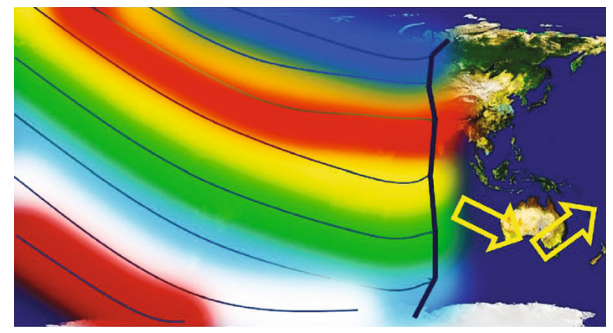

FIGURE 2: Brush lines of 9 satellites in COB $(h=650 \mathrm{~km}, i=60 \mathrm{deg}$, $\varepsilon>10 \mathrm{deg})$.

type of configuration is that it overlaps most satellites and all areas of the Earth), constellation satellites are arranged in a shape of "shape 8" at any moment so that "shape 8 " sweeps bond coverage across the Earth (retrograde orbits from east to west and posigrade orbits from west to east) without gaps as painting the Earth in one revisit time. Brush line of COB (this type of constellation consists of a regular group of 8 planes that form in parallel with each other in perfect harmony with the configuration of the constellation, and the height of each aircraft may vary and the type of satellite may also vary. One advantage of this configuration is that the latitude and longitude coverage is done at the same time and there is user access to all satellites at one time) is the second method that neighboring satellites are arranged with overlap coverage. In this way, small "Brush Lines" of single satellite become a large brush line painting the globe without gaps. Examples of nine satellites in COF and COB for globe coverage are shown in Figures 1 and 2.

Figures 1 and 2 show the brush printing effect of the footprint on the maps. In the COF method, printing completely represents partial of the $\mathrm{COB}$ method; it shows how single footprint of satellite compose a big "Brush Line." In both methods of forming constellations, adjacent satellites can always be visible on the Earth's surface. Accordingly, crosslink ensures that communication between Earth station and constellation satellites will not be interrupted if each satellite is available in view of the Earth station. There are the minimum stations needed to provide continuous communications. Another aspect that will be investigated is close proximity of satellites that makes time to paint the ground. In this analysis, the establishment and optimization of two constellations COF and COB will be discussed. To evaluate the performance of these constellations, comparisons are made by using the results of optimal Walker constellation
TABLE 1: Constellation 1.

\begin{tabular}{lc}
\hline Show & Value \\
\hline$i\left(^{\circ}\right)$ & 80 \\
$h(\mathrm{~km})$ & 1100 \\
$P$ & 3 \\
$F$ & 1 \\
$T$ & 21 \\
\hline
\end{tabular}

TABLE 2: Constellation 2.

\begin{tabular}{lc}
\hline Show & Value \\
\hline$i\left(^{\circ}\right)$ & 65 \\
$h(\mathrm{~km})$ & 750 \\
$P$ & 4 \\
$F$ & 0 \\
$T$ & 28 \\
\hline
\end{tabular}

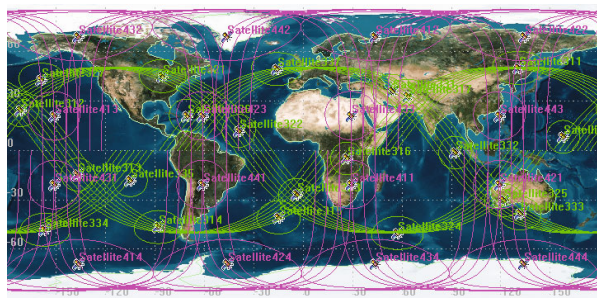

FIgURE 3: The coverage of hybrid constellation.

and genetic algorithms. The results show that, in design of COF constellation, the objectives of the ground coverage can easily be met. Optimization of the revisit time depends on many variables and factors, one of which is the constellation configuration. Due to configuration optimization, other variables will also be optimized. For example, in orbits between $750 \mathrm{~km}$ and $1100 \mathrm{~km}$ high, the orbits are very congested and heavy, so it should be kept in mind that the constellation configuration should be optimized and accurate. Table 1 shows that the altitude of the first constellation is $1100 \mathrm{~km}$, with 21 active satellites in that constellation, and Table 2 shows in the second constellation a height of $750 \mathrm{~km}$, with 28 active satellites. Lower altitude will increase the number of satellites, because the angle of the circle of the satellite coverage on the ground is lowered at a lower altitude. But the quality of the performance is higher and the time for sending and receiving data is less. Also, in the first constellation, due to the fact that the altitude is greater, the number of satellites is lower because the angle of the circle of the satellite coverage is higher. The orbital inclination of 80 degrees in the first constellation is due to the fact that active satellites in the constellation can cover the Arctic and Antarctic regions. The total number of active satellites in this hybrid LEO constellation is 49 satellites, which with an optimal overlap will cover the entire Earth in a revisit. Figure 3 shows simulations of the hybrid LEO constellation. In Figure 3, we see that 


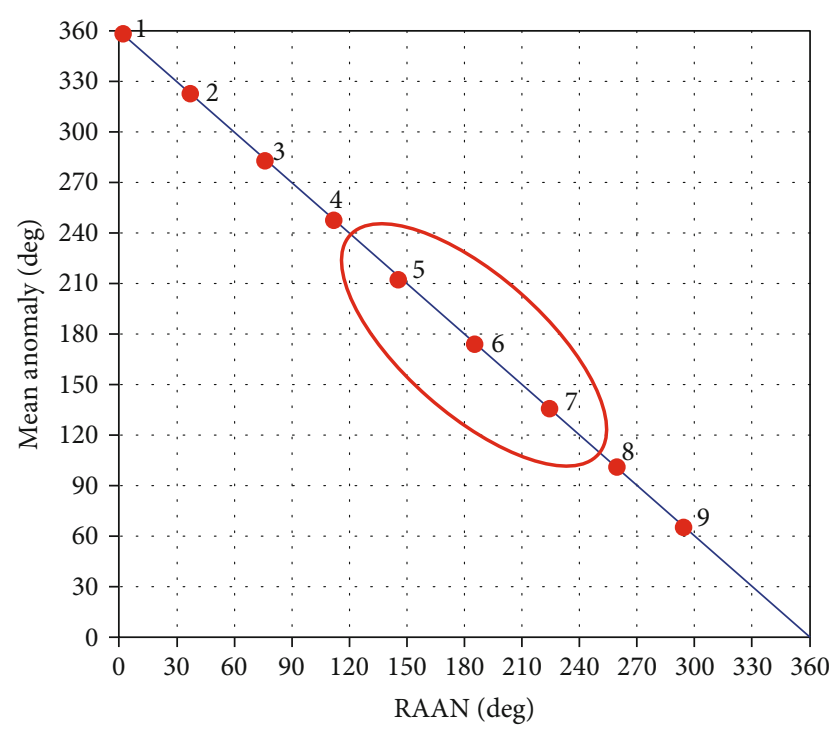

FIgUre 4: RAAN vs. mean anomaly of 9 satellite COF.

the first constellation is shown in red color, and due to higher altitude and 80-degree orbital inclination, all areas of the Antarctic and Arctic are covered. Also, the second constellation is shown in green color which covers areas close to the equator. It is clearly shown in Figure 3 that overlapping of all satellites occurs in constellations 1 and 2. This makes it an ideal global cover across the globe.

\section{RAAN and Mean Anomaly}

Supposed all satellites active in a circular constellation and have the same height and orientation. With selection of the initial values of mean anomaly (MA) and right ascension of ascending node (RAAN), the satellites are tuned into constellation. In Figure 4, these values are shown on a plan. There are 9 active satellites marked by red dots in COF as shown in Figure 4. The satellites active in the constellation are located along the line with slope of -1 .

In inclinations of retrograde, constellation has the same results as line with slope of +1 . Due to the limit of $105 \mathrm{~km}$ altitude, "Reach" crosslinks of 6 satellites are shown in figure with red ellipse curve. This means that each satellite along the blue curve can connect continuously to the other satellites (and line of sight is more than 105 kilometers above the ground). All 9 satellites are located exactly on the curve. In Figure 4, satellite 1 links to 2 , satellite 2 links to 3 , and so on, where the main axis slope is -1 for posigrade orbits and +1 for retrograde orbits. The COF design generates a longer communication time along the main axis.

\section{The Coverage Analysis}

Footprint of a satellite describes the instantaneous coverage on the Earth. Figure 5 shows a satellite at altitude $h$ above the ground where the coverage is limited by elevation angle $\varepsilon$.

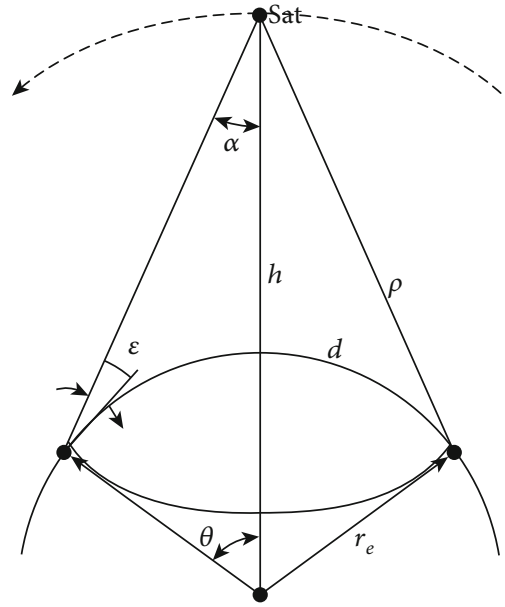

Figure 5: Circle covering a satellite.

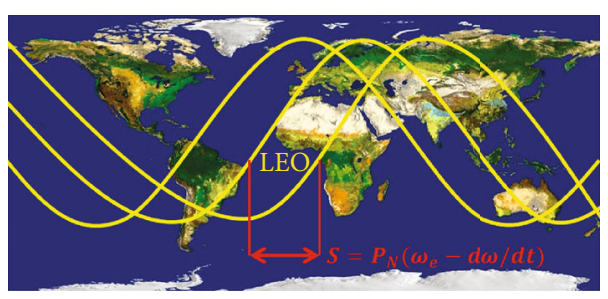

FIgURE 6: The footprints of one LEO satellite.

The following formula is as follows:

$$
\sin \alpha=\cos (\varepsilon+\theta)=\frac{\cos (\varepsilon)}{1+h / R},
$$

where $\alpha$ is the off-nadir angle and $R$ is the radius of the Earth; then, central angle $\theta$ can be calculated by

$$
\sin \theta=\frac{\{\cos \theta-[R / R+h]\}}{\tan \varepsilon} .
$$

Figure 6 shows the footprint movement of the LEO satellites. The revisit curves are moving from east to west and separated by $S$ which is a function of nodal rate $(\Delta \Omega / d t)$, Earth rotational rate $\left(\omega_{e}\right)$, and node period $\left(P_{N}\right) . S$ depends on the inclination and orbit altitude. The details of calculation are in Appendix B. The longitude direction is painted by a ring coverage crossing the equator, because the latitude is enclosed within a large circle as shown in Figure 7. The longitude direction is painted by half-strip $\left(\Delta \lambda^{*}\right)$ passing through the equator line in which it satisfies spherical trigonometry with circular coverage $(\theta)$

$$
\sin i=\frac{\sin \theta}{\sin \Delta \lambda^{*}} .
$$

It is under assumption that the Earth is not spinning and the circle covers overall equator. As time passing, the equator 


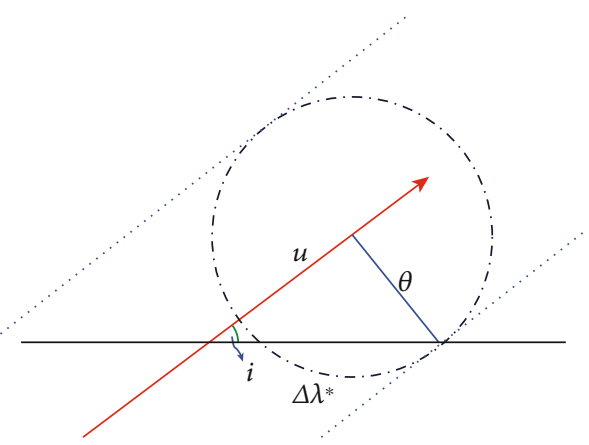

FIgURE 7: The facade of circle coverage on the equator.

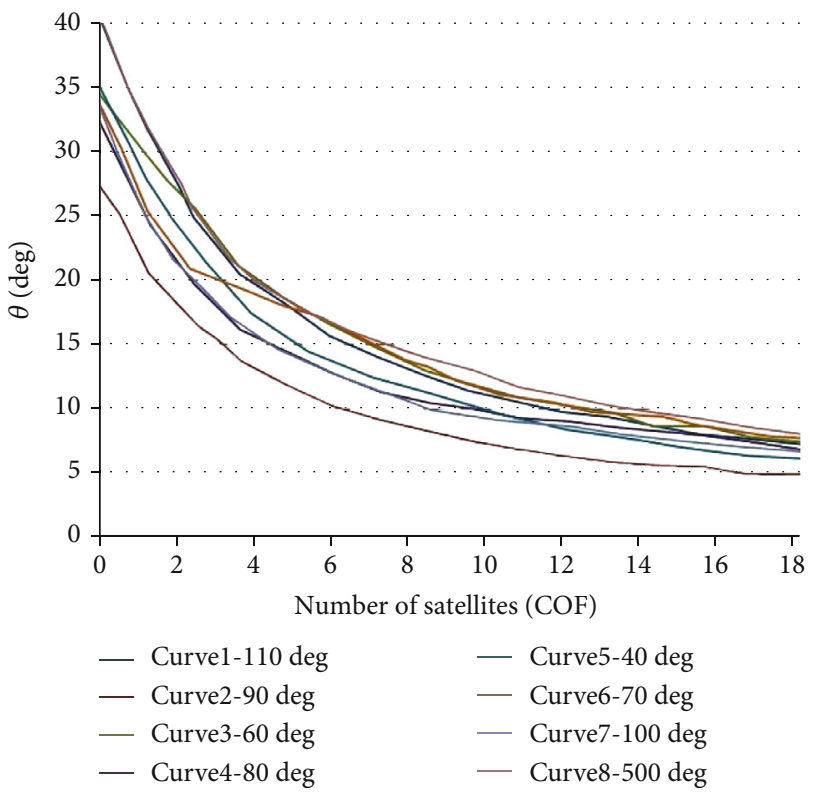

Figure 8: Results of COF constellation.

moves a fraction of $2 u / 2 \pi$ out of orbit. With respect to the plane of the orbit, rotation of the Earth in a revisit is equal to $S$. Therefore, motion $=s(u / \pi)$ and $\tan i=\tan \theta / \sin u$. This amount should either be added to the retrograde stationary band or it should be deducted from the posigrade stationary band to conclude half width of the band in longitude throughout equator.

$$
\begin{aligned}
& a \sin \left(\frac{\sin \theta}{\sin i}\right) \pm\left|s\left(\frac{u}{\pi}\right)\right| \\
& =\Delta \lambda \text { (negative sign for posigrade inclination) }
\end{aligned}
$$

or equivalent

$a \sin \left(\frac{\sin \theta}{\sin i}\right)-\left\{a \sin \left(\frac{\tan \theta}{\tan i}\right)\right\}\left(\frac{s}{\pi}\right)=\Delta \lambda$ (for both modes).

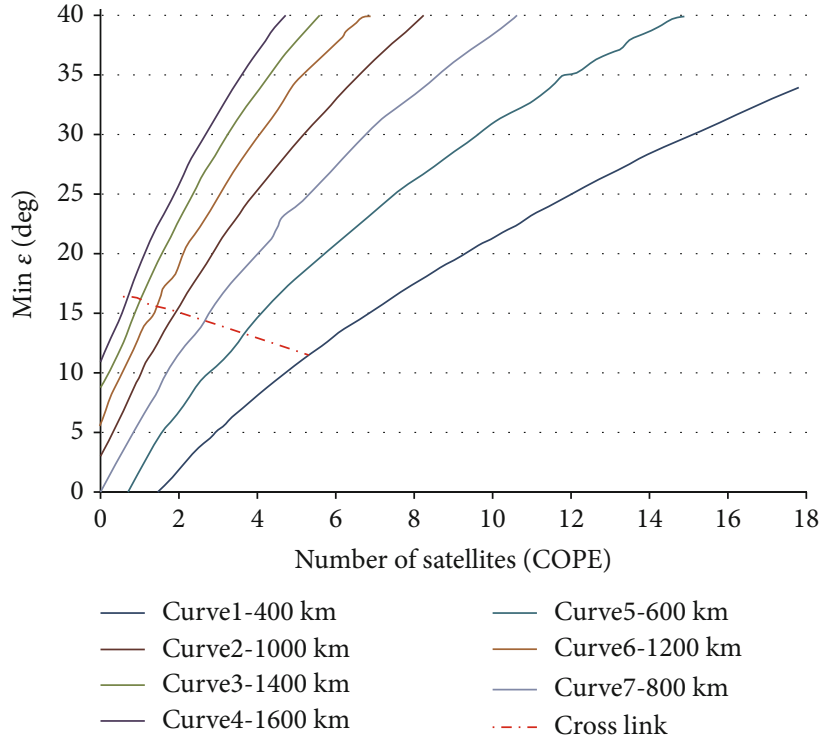

Figure 9: Results of polar COF constellations.

\section{4. "Figure 9" in COF Constellation}

For "Figure 9" constellation, the difference of meaning anomalous $(\Delta M)$ between adjacent satellites is equal to $(\Delta \Omega)$ the difference of RAAN between adjacent satellites.

$\pm \Delta \Omega=\Delta M$ (negative sign for posigrade inclination in COF).

Normally, equator is the hardest latitudinal region because adjacent satellites cover along the equator line must not have spaces and gaps; hence, $\Delta \Omega$ is a key value. Regarding $\Delta M$, $\Delta \Omega$ and $\{S(\Delta M / 2 \pi)+\Delta \Omega\}$ express the difference between the equatorial passages of two satellites. If these settings are equal to $2 \Delta \lambda$, then the paths of the strips are exactly in the vicinity of the equator. So, we have

$$
2 \Delta \lambda=S\left(\frac{\Delta M}{2 \pi}\right)+\Delta \Omega
$$

By combining Equations (8) and (9), $\Delta \Omega$ is obtained by: $\frac{2 \Delta \lambda}{1 \pm S / 2 \pi}=\Delta \Omega$ (negative sign for posigrade inclination)

The COF algorithm is performed as follows:

(1) The values of $h, i$, and $T$ are known

(2) Select elevation angle $(\varepsilon)$

(3) Size of circle coverage $(\theta)$ is obtained by Equation (1)

(4) $S$ is obtained by pairs of Equation (C.1)

(5) $\Delta \lambda$ is obtained by Equation (7) 
TABle 3: $\theta$ requirement for the COF method.

\begin{tabular}{lcccccccc}
\hline Satellites & \multicolumn{8}{c}{ Inclination $(i)$} \\
$(T)$ & 40 & 50 & 60 & 70 & 80 & 90 & 100 & 110 \\
\hline 3 & 33.88 & 39.99 & 33.87 & 32.56 & 31.90 & 26.89 & 31.84 & 31.99 \\
4 & 27.10 & 30.67 & 28.90 & 24.98 & 23.78 & 19.45 & 23.63 & 24.91 \\
5 & 21.50 & 24.49 & 24.69 & 19.49 & 18.80 & 15.73 & 18.56 & 19.86 \\
6 & 17.77 & 19.92 & 20.71 & 18.78 & 15.89 & 12.76 & 15.45 & 18.42 \\
7 & 14.23 & 17.79 & 17.67 & 16.83 & 12.91 & 10.87 & 13.61 & 16.67 \\
8 & 12.31 & 14.69 & 15.71 & 15.43 & 11.40 & 9.94 & 11.93 & 15.87 \\
9 & 11.19 & 12.36 & 13.78 & 13.78 & 10.58 & 8.68 & 10.40 & 14.93 \\
10 & 10.66 & 11.95 & 11.98 & 12.49 & 8.88 & 7.92 & 9.69 & 13.56 \\
11 & 9.45 & 9.56 & 10.53 & 10.66 & 8.49 & 6.84 & 8.78 & 11.84 \\
12 & 8.34 & 8.38 & 9.81 & 9.58 & 8.11 & 5.97 & 8.63 & 10.91 \\
13 & 7.81 & 7.79 & 9.49 & 9.34 & 7.98 & 5.75 & 7.90 & 10.47 \\
14 & 6.94 & 6.98 & 8.57 & 8.80 & 7.67 & 4.98 & 7.56 & 9.89 \\
15 & 6.59 & 6.35 & 7.49 & 8.54 & 6.89 & 4.78 & 7.23 & 8.78 \\
16 & 5.99 & 5.95 & 7.86 & 7.39 & 6.77 & 4.34 & 6.99 & 8.41 \\
17 & 5.82 & 5.57 & 6.81 & 7.09 & 6.42 & 3.89 & 6.67 & 7.79 \\
18 & 5.29 & 5.22 & 6.27 & 6.37 & 5.89 & 3.78 & 6.41 & 6.88 \\
\hline
\end{tabular}

(6) $\Delta \Omega$ is obtained by Equation (10)

(7) $\Delta \Omega$ is obtained by Equation (8)

(8) To determine coverage in one revisit for all satellites on the ground

(9) To completely cover gap, change the elevation angle $(\varepsilon)$ and perform calculations again

(10) To obtain the unique values of $h, i$, and $T$ from the elevation angle

Table 3 shows the results depicted in Figure 8. In this figure, the amount of coverage circle $(\theta)$ is shown on the $y$-axis required for coloring the Earth in one revisit and $x$-axis is the number of satellites. Important results can be seen in the inclination of orbit, but not in the vibrational altitude. It is because altitude is not considered as a parameter. The conclusion is that the volume of coverage circle $(\theta)$ is reduced by increasing the number of satellites.

The performance of a constellation $\theta$ is determined by a certain number of satellites with value $\theta$. To get full coverage on the ground in a revisit, we need a higher elevation angle or lower altitude constellations. As we know, the small size of circler coverage is more optimal and productive than the larger one. According to this indicator, polar constellation is more optimal and efficient because it provides the least value $\theta$ for each satellite. For a certain size of circler coverage, the minimum number of satellites can achieve a complete coverage in a revisit.

Figure 9 shows the minimum elevation angle $(\varepsilon)$ and number of satellites placed in COF polar constellation at different altitudes. The dashed line indicates the crosslinks between any two satellites of constellations at least $105 \mathrm{~km}$ above the Earth.

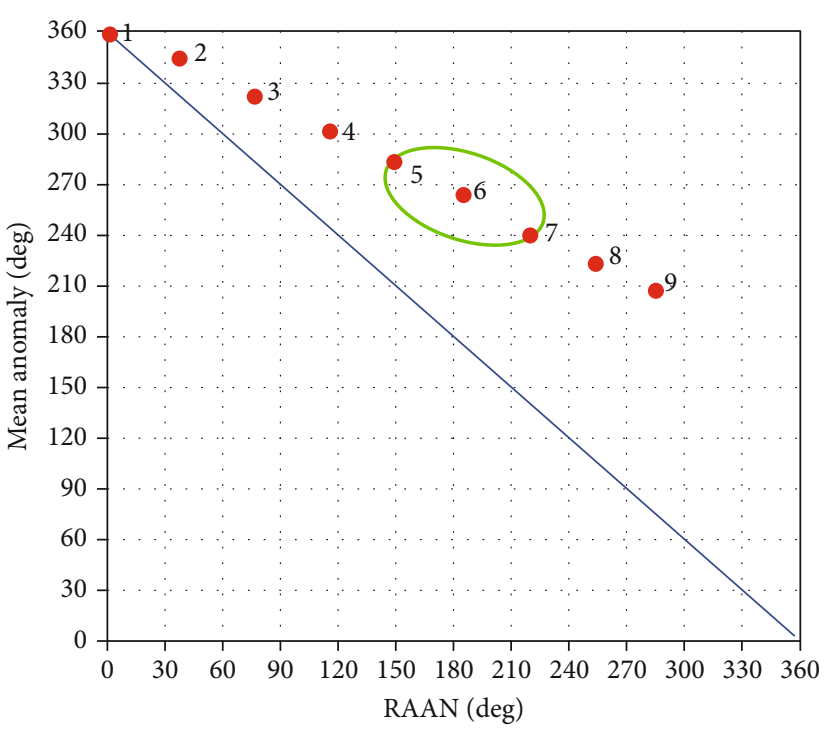

Figure 10: Results for 9 COB satellites.

\section{5. "Brush Line" in COB Constellation}

There are many solutions to move the strip axes of the adjacent satellites together. The COF constellation force can relocate "shape 9" satellites along the longitude. With overlap circle of the coverage, $\mathrm{COB}$ constellation can expand satellites along the longitude reach as far as possible. In this method, individual satellite coverage is converted to large and continuous coverage throughout the land.

In Figure 10, there are 9 active $\mathrm{COB}$ satellites at $650 \mathrm{~km}$ altitude and 60-degree inclination. We cannot calculate the size of circular coverage $(\theta)$ when the elevation angle $(\varepsilon)$ is 10 degrees. The green oval whose central angle of the Earth is $2 \theta$. It indicates that three active satellites in green oval are closer to the $2 \theta$ than other 5 satellites. Simply saying, each active satellite in green oval always has an overlap with other 5 satellite coverages. The green oval can also be placed around any active satellites, in which the main axis is a slope of -1 , and for retrograde orbits, the slope is +1 .

Adjacent satellites that are active in the $\mathrm{COB}$ method are at points of the green oval with a maximum $\Delta \Omega$. The equations For evaluating the values of $\Delta M$ and $\Delta \Omega$ between adjacent satellites in $\mathrm{COB}$ constellation are derived in Appendix C. It guarantees that the coverage of adjacent satellites in the $\mathrm{COB}$ method is always overlapping and expanded as far as possible along the geographic extent.

Below are the steps for implementing the $\mathrm{COB}$ constellation:

(1) Supposed values of $h, i$, and $T$

(2) To select elevation angle $(\varepsilon)$

(3) To get size of circle coverage $(\theta)$ in Equation (1)

(4) Using equations in (A.17) and setting interval $\varphi=2 \theta$ and to get the solution of the oval parameters

(5) Using the equations in Appendix $C$, we can calculate $\Delta M$ and $\Delta \Omega$ 
TABle 4: $\theta$ requirement for the COB method.

\begin{tabular}{lcccccccc}
\hline $\begin{array}{l}\text { Satellites } \\
(T)\end{array}$ & 40 & 50 & 60 & 70 & 80 & 90 & 100 & 110 \\
\hline 3 & 33.99 & 39.91 & 32.83 & 31.11 & 31.91 & 28.97 & 29.99 & 30.83 \\
4 & 27.89 & 31.77 & 28.58 & 23.29 & 21.83 & 21.66 & 22.78 & 23.27 \\
5 & 21.71 & 24.86 & 26.72 & 19.84 & 17.74 & 17.51 & 18.91 & 19.39 \\
6 & 17.78 & 20.69 & 21.50 & 18.67 & 15.92 & 14.48 & 14.98 & 18.54 \\
9 & 11.90 & 13.38 & 14.27 & 14.31 & 9.89 & 9.92 & 9.31 & 14.91 \\
12 & 8.58 & 10.47 & 10.77 & 10.69 & 8.22 & 6.36 & 8.54 & 10.67 \\
15 & 6.37 & 7.63 & 8.61 & 8.48 & 7.41 & 5.41 & 7.73 & 8.87 \\
18 & 5.44 & 6.58 & 6.49 & 6.55 & 6.62 & 4.26 & 6.29 & 6.44 \\
\hline
\end{tabular}

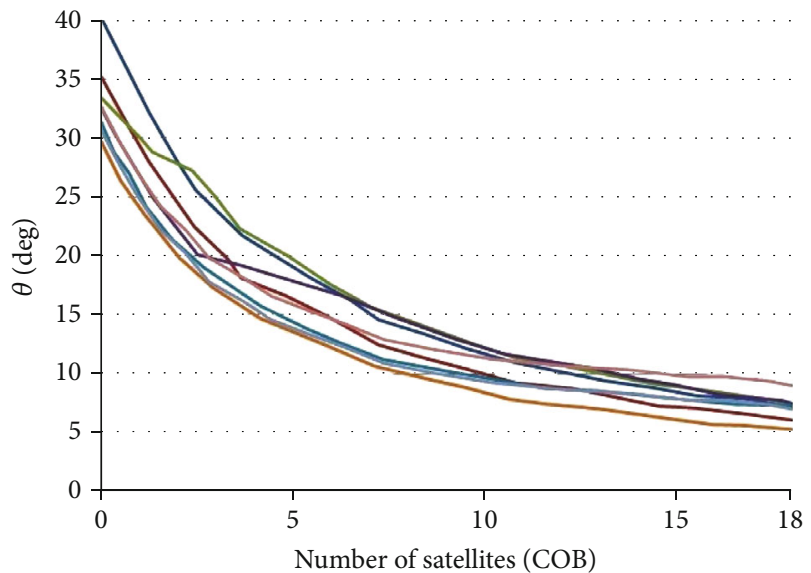

$$
\begin{array}{ll}
\text { - Curve1-50 deg } & \text { - Curve5-80 deg } \\
\text { - Curve2-40 deg } & \text { Curve6-90 deg } \\
\text { - Curve3-60 deg } & \text { Curve7-100 deg } \\
\text { - Curve4-70 deg } & \text { - Curve8-110 deg }
\end{array}
$$

Figure 11: Results for COB.

(6) To determine coverage of all satellites in one revisit

(7) To completely cover the gaps, change the elevation angle $(\varepsilon)$, and perform the calculations again

(8) To obtain the unique value of $h, i$, and $T$ from the elevation angle

Table 4 and Figure 11 show the coverage results of $\mathrm{COB}$ satellite with parameters $i, t$, and $h$ obtained by the steps above. In Figure 11, $y$-axis presents the size of coverage or central angle $(\theta)$ in a revisit time and $x$-axis presents the number of active satellites. It can be seen that vibration results go with the inclination of orbit, but not with the altitude, and with increasing number of active satellites in the constellation. The polar constellations are more efficient in consistence and continuous, even though they are not as much as COF constellations. Polar constellations provide the least value $\theta$ for all satellites. For a certain size of coverage, polar constellations can provide a complete coverage with the minimum number of satellites in one revisit time.

Figure 12 shows the results of polar $\mathrm{COB}$ constellations with coverage size or central angle $(\theta)$ respecting to elevation
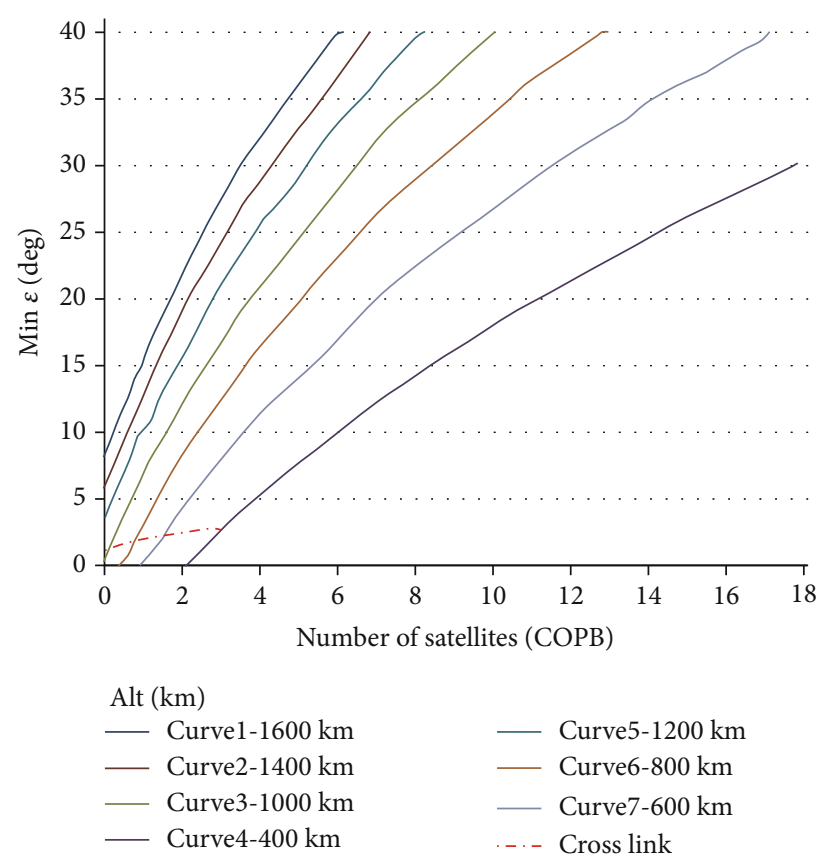

FIgURE 12: Results for polar COB.

TABLE 5: COF.

\begin{tabular}{lc}
\hline Show & Value \\
\hline$i\left(^{\circ}\right)$ & 50 \\
$h(\mathrm{~km})$ & 1000 \\
$P$ & 8 \\
$F$ & 1 \\
$T$ & 48 \\
\hline
\end{tabular}

angle $(\varepsilon)$. Using this chart, one can find out how many satellites should be applied for the COB polar constellation in a revisit time with the minimum elevation angle. The dashed line indicates the crosslink between the satellites whose altitudes remain at least $105 \mathrm{~km}$. Comparing to the COB constellations, the elevation angles of polar COB constellations are less for the same crosslink and require more satellites. So, we can conclude that $\mathrm{COB}$ polar constellations are more optimal and efficient. Although the results indicate that the efficiency of polar $\mathrm{COB}$ constellations is better, there may be certain conditions that the continuous coverage of crosslink goes towards global returns. Nonpolar COB may be more optimal and efficient for these types of applications.

Optimization of the revisit time depends on many variables and factors, one of which is the constellation configuration. Due to configuration optimization, other variables will also be optimized. In this paper, the simulation is carried out and the number of satellites and other variables is taken into account. So, let us assume that the initial elevation angle is $5^{\circ}$, there is a constellation named by COF. Table 5 shows the results of the simulation parameters for optimal design of the LEO constellation. The coverage of constellation is shown in Figure 13. COF is 2-fold coverage or greater and 


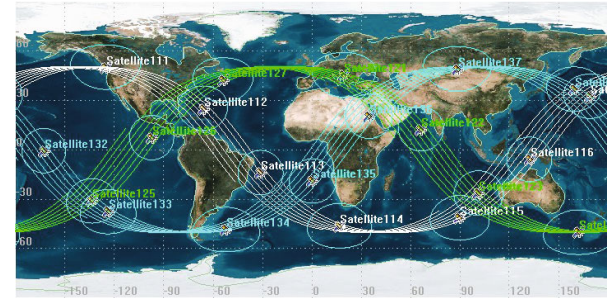

FIgURE 13: The coverage of COF.

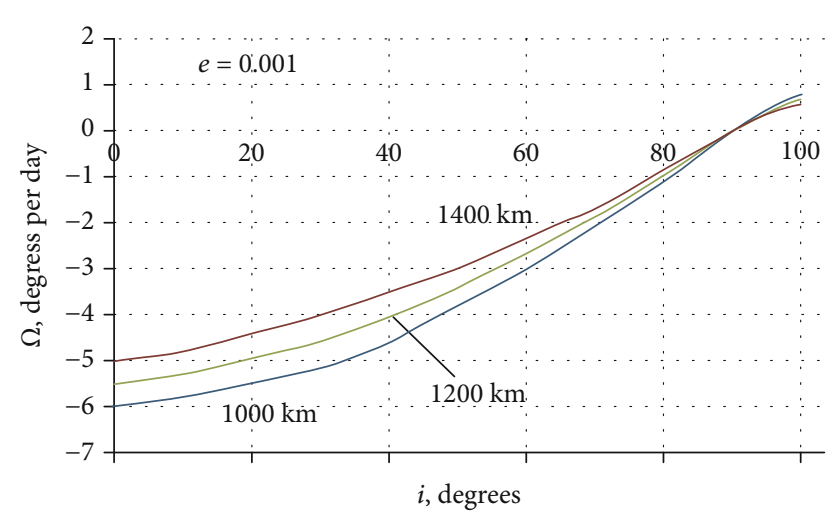

FIGURE 14: Orbital precessional rate vs. inclination angle and orbital altitudes to select the optimum set of parameters.

in the equator areas are 3-fold coverage which can almost realize global communication. Besides, a small area is 1-fold or 3-fold coverage, and some areas are 3-fold coverage. Also shown in Figure 14 are variations and proportions of orbital precessional rate and inclination angle and orbital altitudes to select the optimum set of parameters.

\section{More Results of MRT in Different Constellations}

In order to compare the performance of GA and Walker constellations mentioned above, let us take the examples of optimizing MRT values in Reference [3]. Considering the elevation angle $=0 \mathrm{deg}$, there are 4 satellites at altitude of $700 \mathrm{~km}$ and 3 satellites at altitude of $1100 \mathrm{~km}$.

Using Equation (1), for 4 satellites at altitude of $1100 \mathrm{~km}$, the central angle of coverage circle is calculated by $\theta=31.47$ degrees and elevation angle is 0 degree. According to Figure 8 , the better performance of 4 active satellites in COF happens in 90-degree inclination, not in 100 degree and more, or in 80 and less. In the COF method described above, we make elevation angle as zero, inclination as $90 \mathrm{deg}$, and altitude as $1100 \mathrm{~km}$. The efficiency happens when $-\Delta M=\Delta \Omega=68$ degrees. In other words, the mean anomaly and RAAN difference between the adjacent satellites are -68 degrees and +68 degrees, respectively. Figure 12 shows the values of MRT as a function of latitude at minimum elevation angle $=0$ for COF, Walker, and GA orderings.

The MRT curves of COF, GA, and Walker constellations are similar below 20 degrees latitude. MRT of GA and COF

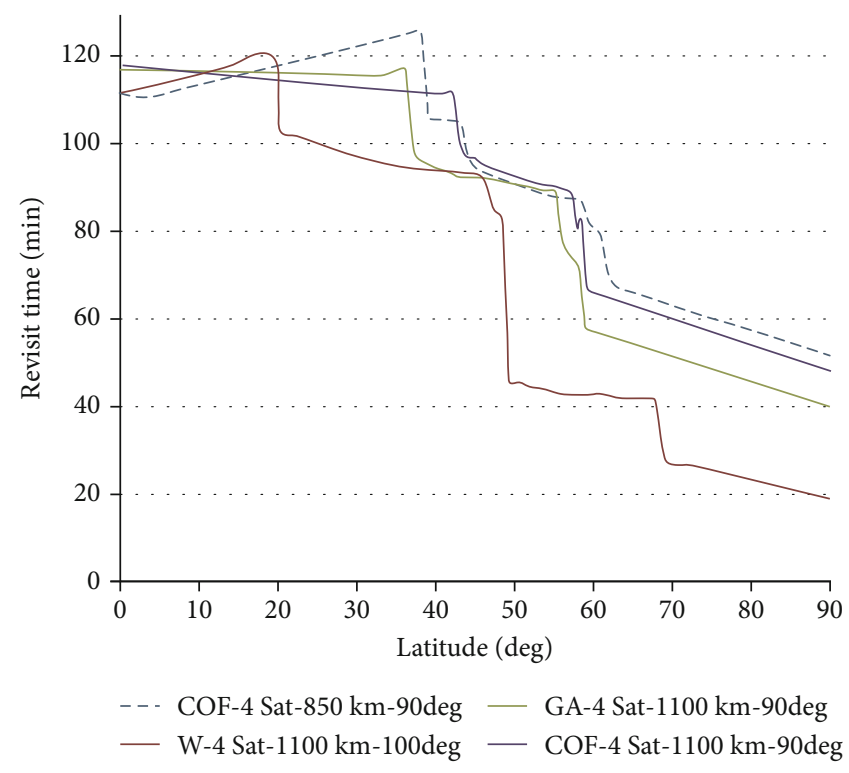

FIGURE 15: Compare MRT variations for 4 satellite constellations.

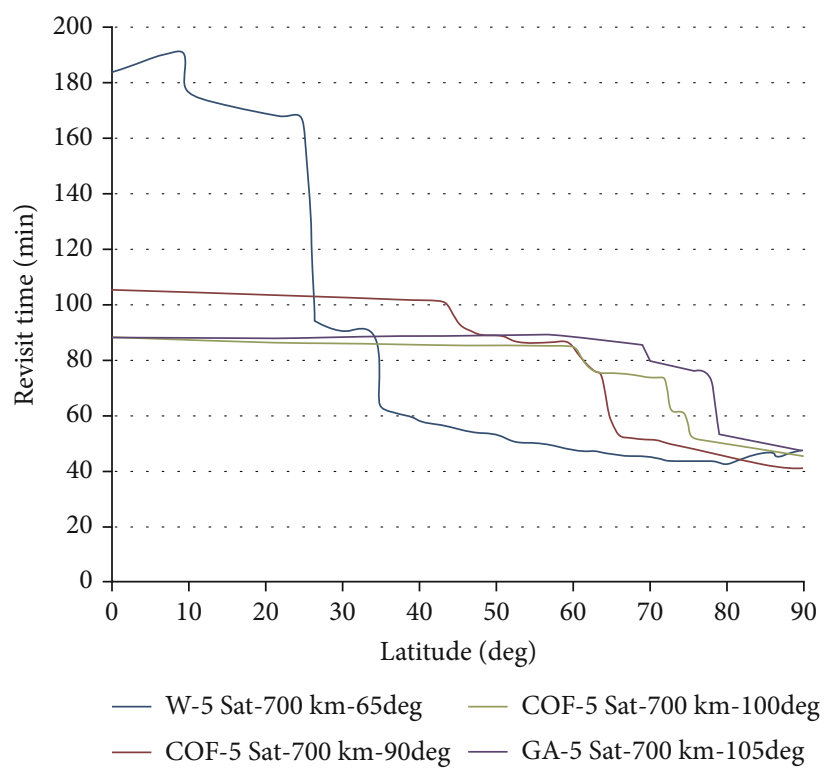

FIgURE 16: MRT variations for 5 satellite constellations.

models are larger than Walker at the higher latitudes. According to Figure 9, for 4 active satellites, the optimal solution of COF is obtained when altitude is $850 \mathrm{~km}$ and elevation angle is zero and $-\Delta M=\Delta \Omega=60.4$ degrees. It is shown by a dashed line in Figure 15.

Figure 16 shows 5 satellites of COF, Walker, and GA constellations in which elevation angle $=0$ degree, central angle $\theta=25.7$ degrees, and all altitudes are $700 \mathrm{~km}$; the inclination changes from 65 to 105 . In retrograde inclinations, the direction of moving satellites is opposite of the Earth's rotation. The curves are obtained as $-\Delta M=\Delta \Omega=49$ degrees and inclination is 100 degrees in Reference [3].

It is also shown that the results of 5 satellites in COF are close to the GA method. Clearly, coverage of the COF in latitude below 50 degrees is better than GA constellation that 


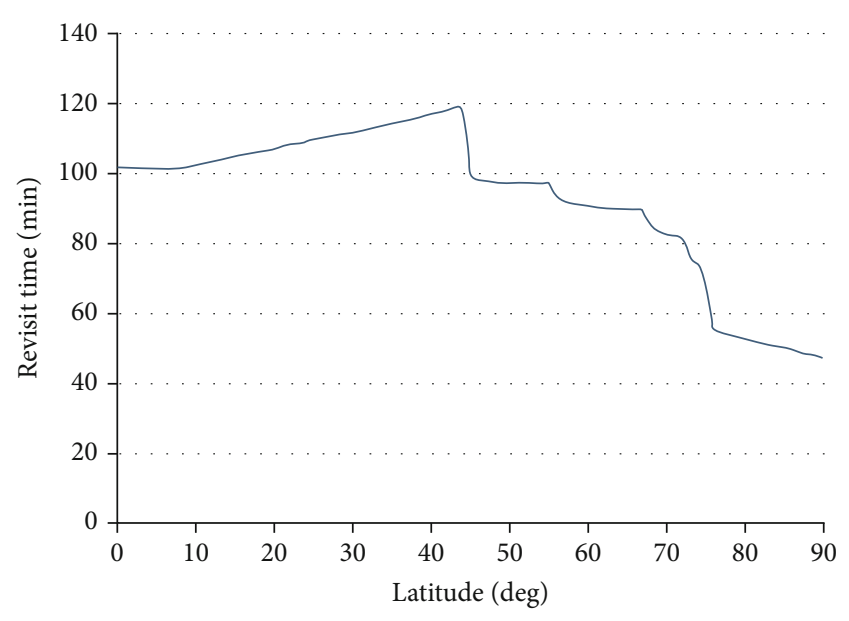

FIGURE 17: Results for 6 COF satellites at $650 \mathrm{~km}$.

extends upper latitude. Walker constellation appears better coverage in latitude below 30 degrees. The coverage of 5 satellite constellations active at $700 \mathrm{~km}$ altitude presents better performance than 4 satellite constellations at $1000 \mathrm{~km}$ altitude in more revisit time.

\section{More Results in COF Constellations}

In this sample, 6 polar COF satellites at $650 \mathrm{~km}$ altitude with elevation angle $>10^{\circ}$ are applied to cover the entire Earth. According to Figure 8, central angle $\theta=14.6$ degrees, latitude is 12 degrees, and 6 satellites are able to cover the entire Earth in one revisit. In the algorithm of Section $4,-\Delta M=\Delta \Omega=$ 31.3 degrees that indicates a decrease of 31 degrees for optimizing the altitude of crosslink. Using Equations (A.4) and (A.1) and $-\Delta M=\Delta \Omega=31$ degrees, we conclude that the minimum height required for adjacent satellites in the constellation to provide continuous communications must be 105 kilometers. The maximum range of crosslink is about 5200 kilometers. Displayed in Figure 17 for six satellites in the COF method, the minimum elevation angle is 12 degrees, if the maximum MRT is obtained. The MRT is slightly bigger than the orbital period that is 97 minutes. All the satellites in constellation can contact to the Earth station any time; each of them is accessible to the Earth station whatever it is visible or not, because there are crosslinks in the constellation.

Most of the time, satellites are moving at high latitudes, especially in 90 degrees inclination; thus, the best position for Earth stations to communicate with satellites is near the pole. In this example, if the elevation angle is greater than 10 degrees, the Earth stations near the pole are in Arctic or Antarctic, where the locations are McMurdo (77.7 S, 168.2 E) and Thule $(76.5 \mathrm{~N}, 68.7 \mathrm{~W})$. The simulation results indicate that satellites can connect the Earth station for more than $83 \%$ of the time, as shown in Figure 18; during a day, at least one satellite in the constellation can connect McMurdo or Thule station.

In Figure 19, within footprint of satellites and at least one of two active satellites is available to the Earth station at any moment, and the revisit time equals to 104 minutes. In the simulation, there are small spots (cavity) in which there is

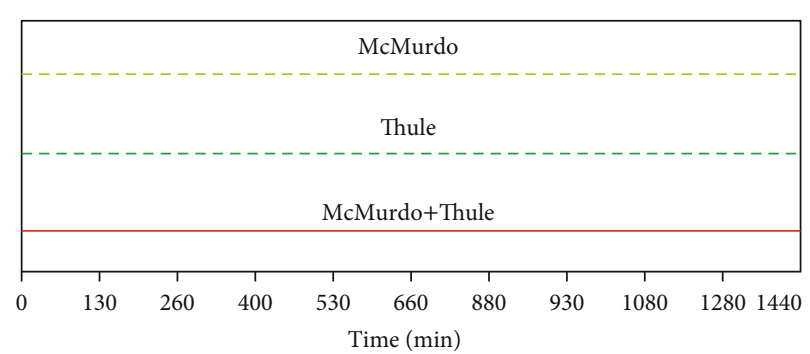

FIGURE 18: Sample 1: the amount of communications in one day for 6 COF satellites.

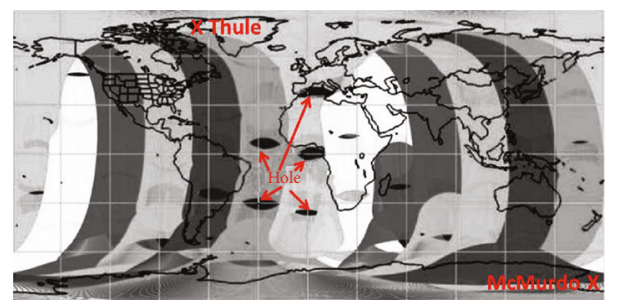

FIGURE 19: Ground coverage results by 6 COF satellites in 1 revisit time.

no connection to the Earth stations. Because these spots are inside adjacent coverage at different times, different satellites may cover the entire Earth and contact a land station; we conclude that the entire Earth can be covered in each revisit, and use two Earth stations simultaneously thru ISL. The simulation of coverage routine shows that the land coverage is different from one revisit to a subsequent revisit.

\section{Conclusion}

In an optimal constellation, satellite footprint expands in the pattern of a brusher across the Earth. A new method is proposed in this paper that active satellites are located in "Figure 9" pattern or become a great brusher to cover the entire Earth. This design makes the constellation coverage without any cavities in low latitude. If the inclination of satellites is more than 90 degrees, the Earth's poles will also be covered. At suitable altitude, the maximum revisit time may be 90 to 130 minutes, so that satellites can coverage the entire Earth in one revisit.

In the design of $\mathrm{COB}$ and COF constellations, one can choose the elevation angle, to define the coverage level, and choose the altitude to determine the central angle of coverage. In order to completely cover the ground in a revisit, designers can use the charts, tables, and figure provided in this report to choose number of satellites in the constellation and inclinations, further to determine initial values of the mean anomaly (MA) and right ascension of ascending node (RAAN).

The constellations are optimized by the genetic algorithm. Through the simulation, MRT is optimized in a revisit, the design and process of COF constellation are more convenient and efficient than COB. So, it concludes that COF constellations are more efficient and more practical. By using charts, figures, and tables, people can understand how much impact of the initial parameters on final results. 


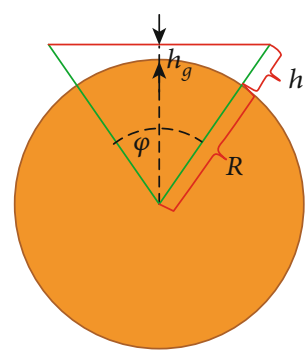

FIGURE 20: Minimum height range for the crosslink.

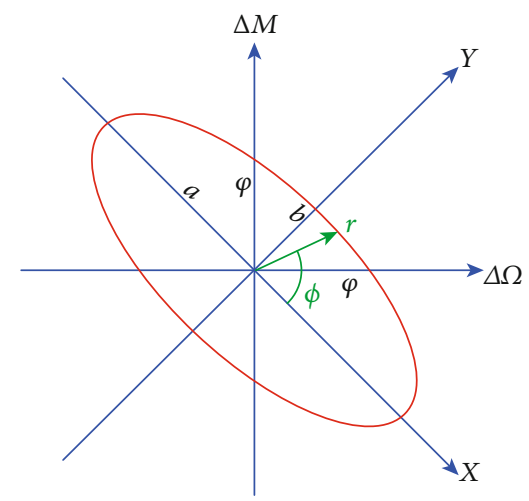

FIgURE 21: The best range to create crosslinks.

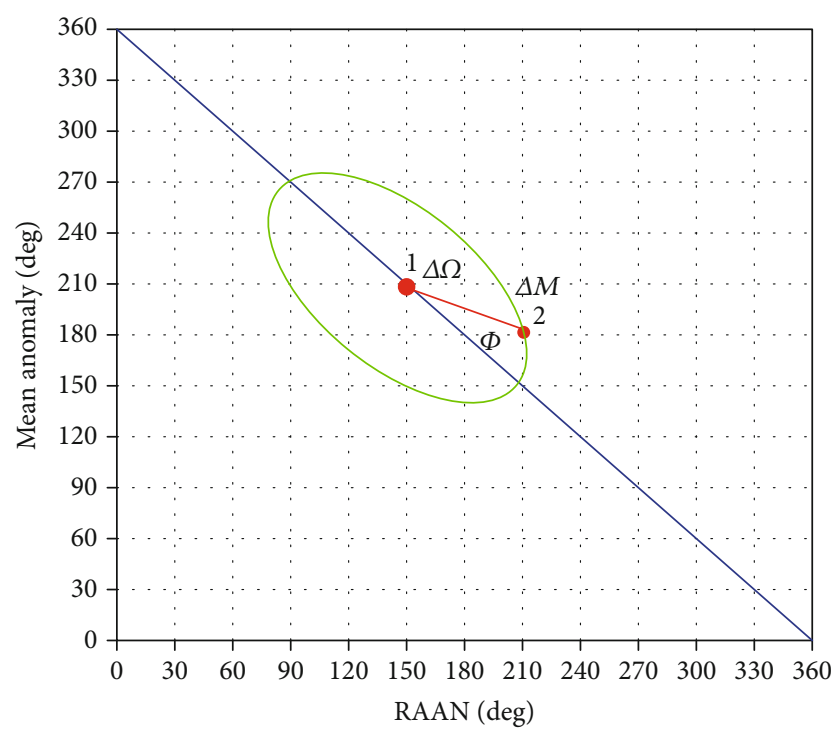

FIgURE 22: The most effective range of crosslinks between two neighboring satellites.

The satellites in COF and COB constellations have the advantages of maintaining the inter satellite links (ISL) continuous communication. Meanwhile, the satellites in both constellations are able to communicate with the Earth station in blind spots, because Earth station can connect any other satellites thru the ISL of available and visible satellites. Thus, less Earth stations are needed to communicate with satellites.

\section{Appendix}

\section{A. Calculations of the Crosslink in Ellipse}

The mean anomaly (MA) and right ascension of ascending node (RAAN) are values that determine the basic configuration of a constellation. Taking the example in Figure 4, there are 9 satellites in the constellation coordinated with the line of slope $=-1$ and RAAN $=-\mathrm{MA}$; all active satellites in the constellation have the same inclination. The number 6 satellite indicates that it is reaching the crosslink and communication area. Figure 20 represents required height $(\mathrm{hg})$ of satellite crosslink and the coverage area.

$\varphi$ represents central angle of the optimal coverage area where Earth station can communicate with each satellite. As long as the value of $\varphi$ is equal to (A.2), all the satellites can communicate the Earth station within coverage. Figure 21 is the magnification of Figure 4 in which two parameters $\Delta \Omega$ and $\Delta M$ meet the requirements of $\varphi$ and $h=650 \mathrm{~km}, i=60^{\circ}$ and $h g=105 \mathrm{~km}$.

For a satellite to communicate in crosslink, we obtain the biggest value of Earth's central angle $(\varphi)$ in terms of $h$ and $h g$.

$$
\frac{R+h_{g}}{\cos }\left(\frac{\varphi}{2}\right)=(R+h)
$$

where $R$ represents radius of the Earth.

The ground pitch between two adjacent satellites is determined by difference $\Delta \Omega$ and $\Delta M$ as reference [6], and the maximum value $\varphi$ is expressed as follows:

$$
\cos ^{2} \frac{\varphi_{\max } / 2}{\cos ^{2}}\left(\frac{\alpha}{2}\right)=\cos ^{2}\left(\frac{\Delta f}{2}\right),
$$

where $\alpha$ represents the angle between two orbital planes, so

$$
\begin{array}{r}
\cos \alpha-\sin ^{2} i \cos \Delta \Omega=\cos ^{2} i, \\
\Delta f+2 \tan ^{-1}\left(-\tan \left(\frac{\Delta \Omega}{2}\right) \cos i\right)=\Delta M .
\end{array}
$$

To obtain the partial axes of oval in elliptic equation,

$$
1=\frac{x^{2}}{a^{2}}+\frac{y^{2}}{b^{2}}
$$

suppose ellipse has a crossing point

$$
\frac{\varphi}{\operatorname{sqrt}(2)}=x=y
$$

the semiminor axis $b$ is obtained by:

$$
\frac{0.5 a^{2} \varphi^{2}}{a^{2}-0.5 \varphi^{2}}=b^{2} \text {. }
$$


If major axis is in the ellipse,

$$
c=\Delta M=-\Delta \Omega .
$$

For semimajor axis,

$$
2 c^{2}=a^{2} .
$$

From (A.2), (A.3), and (A.4) to find out $c$, replacing (A.3) and (A.8) by (A.2), $\alpha$ will be deleted:

$$
\left(1-\sin ^{2}\left(\frac{\mathcal{c}}{2}\right) \sin ^{2} i\right) \cos ^{2}\left(\frac{\Delta f}{2}\right)=\cos ^{2}\left(\frac{\varphi}{2}\right) .
$$

Then, replace (A.6) with (A.4):

$$
\left(1+\tan ^{2}\left(\frac{c}{2}\right) \cos i\right) \tan \left(\frac{\Delta f}{2}\right)=\tan \left(\frac{c}{2}\right)(1-\cos i) .
$$

Remove $\Delta f / 2$ in (A.10) and (A.11) by using $\cos ^{2}=1 /(1+$ $\left.\tan ^{2}\right)$. Then,

$$
\begin{aligned}
\tan ^{2}\left(\frac{c}{2}\right) & =x, \\
\cos i & =g, \\
\cos \left(\frac{\varphi}{2}\right) & =h .
\end{aligned}
$$

Then, a third-order equation is obtained as follows:

$$
\begin{array}{r}
x^{3}\left(g^{4}-g^{2} h^{2}\right)+x^{2}\left(2 g^{3}+g^{2}-2 g^{2} h^{2}-h^{2}\right) \\
+x\left(g^{2}-g^{2} h^{2}+2 g-2 h^{2}\right)+\left(1-h^{2}\right)=0, \\
x^{3}+p x^{2}+q x+r=0 .
\end{array}
$$

With cubic solution,

$$
\begin{aligned}
A & =\frac{3 q-p^{2}}{3}, \\
B & =\frac{2 p^{3}-9 p q+27 r}{27}, \\
\text { Radical } & =\frac{B^{2}}{4}+\frac{A^{3}}{27} .
\end{aligned}
$$

In the case of radicals $<0$,

$$
x=m \cos \gamma-\left(\frac{p}{3}\right),
$$

where $m=2 \operatorname{sqrt}(-A / 3) \cos (3 \gamma)=3 B /(A m) . c$ is given by:

$$
c=2 a \tan (\operatorname{sqrt}(x)) .
$$

In terms of $h$ and $h g$, we obtain the maximum of Earth's central angle $(\varphi)$.
From Equation (A.1), we find the semimajor axes $(a)$ and semiminor axes $(b)$.

If $u=\cos i$ and $v=(\varphi / 2)$.

By solving the equations, we have the following:

$$
\begin{aligned}
& d=u^{4}-u^{2} v^{2}, \\
& p=\frac{2 u^{3}+u^{2}-2 u v-v^{2}}{d}, \\
& q=\frac{u^{2}-u v^{2}+2 u-2 v^{2}}{d}, \\
& r=\frac{1-u^{2}}{d}, \\
& A=\frac{3 q-p^{2}}{3}, \\
& B=\frac{2 p^{3}-9 p q+27 r}{27}, \\
& m=2\left(\frac{-A}{3}\right)^{0.5}, \\
& \gamma=\left(\frac{1}{3}\right) a \cos \left(\frac{3 B}{m A}\right), \\
& x=m \cos \gamma-\left(\frac{p}{3}\right), \\
& c=2 \operatorname{atan}(\operatorname{sqrt}(x)), \\
& a^{2}=2 c^{2}, \\
& b^{2}=\frac{(1 / 2) a^{2} \varphi^{2}}{a^{2}-(1 / 2) \varphi^{2}} .
\end{aligned}
$$

Considering $\Phi$ as the $x$-axis of the ellipse, we have "infinity" points as follows:

$$
\begin{aligned}
r^{2} & =\frac{a^{2} b^{2}}{a^{2} \sin ^{2} \Phi+b^{2} \cos ^{2} \Phi}, \\
\Delta \Omega & =r \cos (\Phi-45), \\
\Delta M & =r \sin (\Phi-45) .
\end{aligned}
$$

In retrograde orbits, the slope of the main axis is +1 .

\section{B. How to Determine the Motion of Satellite Related to the Ground}

In Figure 6, parameter $S$ represents shifting longitude of satellite footprint in a revisit. Due to rotation of the Earth, the orbital plane rotates in RAAN as $J_{2}$.In an orbit, inclination is $i$, eccentricity is $e$, and semimajor axis is $a . S$ is obtained through the following equations:

$P_{0}=2 \pi \operatorname{sqrt}\left(\frac{a^{3}}{\mu}\right)($ Keplerian period $)$,

$L_{1}=1.5 J_{2}\left(\frac{R}{p}\right)^{2}\left(R\right.$ is the Earth radius and $\left.p=a\left(1-e^{2}\right)\right)$, 


$$
\begin{aligned}
L_{2} & =1.5 \sin ^{2}(i), \\
L_{3} & =\operatorname{sqrt}\left(1-e^{2}\right), \\
L_{4} & =2.5 \sin ^{2}(i), \\
P_{N} & =P_{0}\left[1-L_{1}\left[\left(1-L_{2}\right) L_{3}+2-L_{4}\right]\right] \text { (nodal period), } \\
\Delta \Omega & =-2 \pi L_{1} \cos i(\text { nodal regression per revisit), } \\
S & =\omega_{e} P_{N}-\Delta \Omega\left(\omega_{e} \text { is the Earth rotation rate }\right) .
\end{aligned}
$$

\section{How to Obtain Parameters of COB}

The satellites covering ground by footprints must be adjacent to each other. According to the central angle of the coverage, the distance between two neighboring satellites should be less than $2 \theta$. In the COB method, the maximum amount of RAAN is set as shown in Figure 22. Two satellites 1 and 2 should be spaced by $2 \theta$ in order to reach the focal point. In this way, small brush lines turn into a large brush coverage.

Given parameters are as follows: $i(\mathrm{deg})$, elevation angle, $(\varepsilon) h(\mathrm{~km})$.

We can calculate the coverage in footprint of the satellite as follows:

$$
\begin{aligned}
&\left(1+\frac{h}{R}\right) \cos (\theta+\varepsilon)=\cos \varepsilon(R \text { is the Earth radius }), \\
& \varphi=2 \theta(\text { it means that the "reach" range is } \\
&\text { twice of satellite footprint }) .
\end{aligned}
$$

For continuous overlap of the brushes, the maximum angular distance between the adjacent satellites is $\varphi$; we have desired oval for satellite 2 by using Equation (A.17) in Appendix A.

The elliptic equation for "reaching" the coverage area is as follows:

$$
1=\frac{x^{2}}{a^{2}}+\frac{y^{2}}{b^{2}}
$$

Take differential of $x$ as follows:

$$
-b^{2} \frac{x}{a^{2} y}=\frac{d y}{d x} .
$$

Let two satellites 1 and 2 be far as possible and $\Delta \Omega$ reach the largest value; at this point, the slope is 45 degrees relative to the main axis of the ellipse.
Then, $-1=d y / d x($ or +1 for $i>90 \mathrm{deg})$; solve for $y$ :

$$
y=\left(\frac{b^{2}}{a^{2}}\right) x .
$$

Use the ellipse equation to solve $x$ and $y$

$$
\begin{aligned}
& x=\operatorname{sqrt}\left(\frac{1}{\left(1 / a^{2}\right)+\left(b^{2} / a^{4}\right)}\right), \\
& y=\operatorname{sqrt}\left(\frac{b^{4}}{a^{2}+b^{2}}\right) .
\end{aligned}
$$

Put $x, y$ in equations of satellite 2 .

For $\Delta \Omega, \Delta M$,

$$
\begin{aligned}
\Phi & =a \tan \left(\frac{y}{x}\right), \\
r^{2} & =x^{2}+y^{2} \\
\Delta \Omega & =r \cos (45-\Phi), \\
\Delta M & =-r \sin (45-\Phi)(\text { and positive sign if } i>90) .
\end{aligned}
$$

Equation (A.17) can be used for all posigrade and retrograde orbits.

\section{Nomenclature}

COF: Constellation of Figure 9

COB: Constellation of brush line

$R: \quad$ Earth radius

$\omega_{e}: \quad$ Earth rotation rate

$T / P / F$ : Constellation parameters (satellites/planes/interplane phasing)

a: $\quad$ Orbit semimajor axis

$h$ : Orbit altitude

$i$ : Orbit inclination

$e: \quad$ Orbit eccentricity

$p: \quad$ Orbit semilatus rectum, $p=a\left(1-e^{2}\right)$

$u$ : $\quad$ Satellite argument of latitude

$\Delta M: \quad$ Mean anomaly difference between adjacent satellites

$\Delta \Omega$ : $\quad$ Right ascension of ascending node (RAAN) difference between adjacent satellites

$\varepsilon$ : $\quad$ Ground elevation angle of coverage circle

$\theta$ : $\quad$ Earth central angle of coverage circle

$\alpha: \quad$ Off-nadir angle of coverage circle

$S$ : $\quad$ Longitude shift of ground track per rev

$\Delta \lambda$ : Half-width of longitude swath at equator

$\varphi$ : $\quad$ Earth central angle of crosslink "reach" between satellites

$h_{g}$ : Grazing altitude of the crosslink line of sight between satellites

$\gamma$ : $\quad$ Angle between the normals to two orbit planes (plane change angle)

MRT: Max revisit time

GA: Genetic algorithms 
ART: Average revisit time

MA: Mean anomaly

RAAN: Right ascension of ascending node.

\section{Data Availability}

We have included the necessary data in the article. The tables and figures have included the necessary data in the article.

\section{Conflicts of Interest}

The authors declare that they have no conflicts of interest.

\section{References}

[1] E. George, "Optimization of satellite constellations for discontinuous global coverage via genetic algorithms," in AAS 97621, AAS/AIAA Astrodynamics Specialist Conference 1997, Sun Valley, ID, August4-7, 1997, Advances in the Astronautical Sciences Series, Vol. 97, pp. 333-347, Univelt Inc., San Diego, 1998.

[2] O. Abdelkhalik and D. Mortari, "Orbit design for ground surveillance using genetic algorithms," Journal of Guidance, Control, and Dynamics, vol. 29, no. 5, pp. 1231-1235, 2006.

[3] Z. Peng and S. Kohani, "The performance of the constellations satellites based on reliability," The Journal of Space Safety Engineering, vol. 4, no. 2, pp. 112-116, 2017.

[4] D. Casanova, M. Avendano, and D. Mortari, "Optimizing flower constellations for global coverage," in AIAA/AAS Astrodynamics Specialist Conference, Minneapolis, MN, USA, August 2012.

[5] M. P. Ferringer and D. B. Spencer, "Satellite constellation design tradeoffs using multiple-objective evolutionary computation," Journal of Spacecraft and Rockets, vol. 43, no. 6, pp. 14041411, 2006.

[6] M. L. Marcus and R. J. Sedwick, "Low Earth orbit debris removal technology assessment using genetic algorithms," Journal of Spacecraft and Rockets, vol. 54, no. 5, pp. 1110-1126, 2017.

[7] W. Mason, V. Coverstone-Carroll, and J. Hartmann, "Optimal Earth orbiting satellite constellations via a Pareto Genetic Algorithm," in AIAA/AAS Astrodynamics Specialist Conference and Exhibit, Boston, MA, USA, August 1998. 


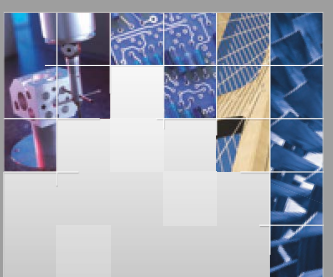

\section{Enfincering}
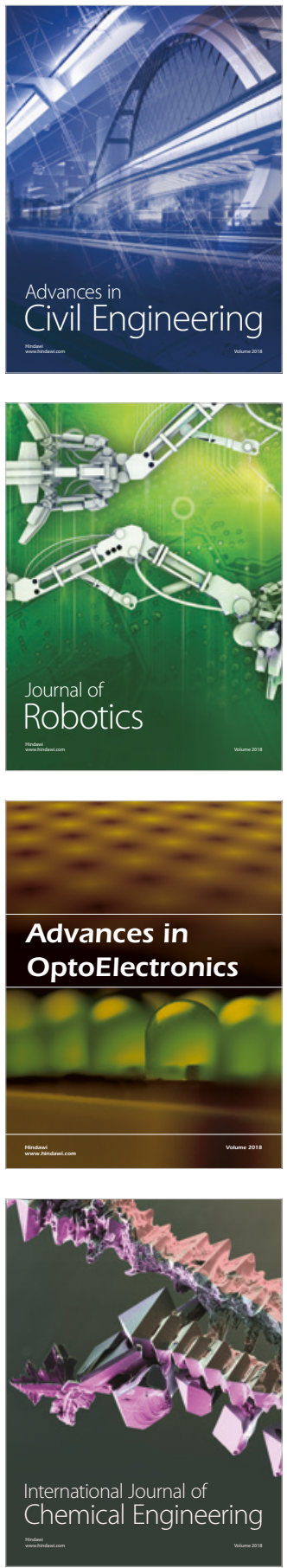

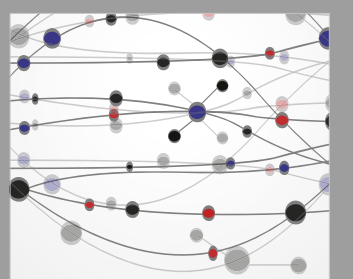

\section{Rotating \\ Machinery}

The Scientific World Journal

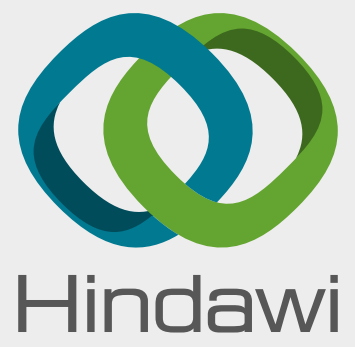

Submit your manuscripts at

www.hindawi.com
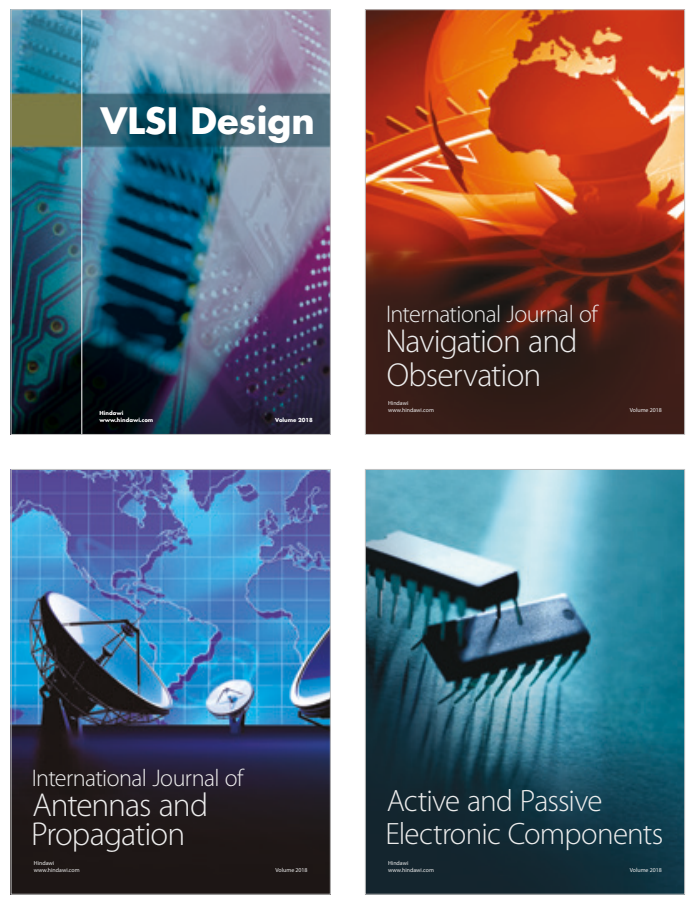
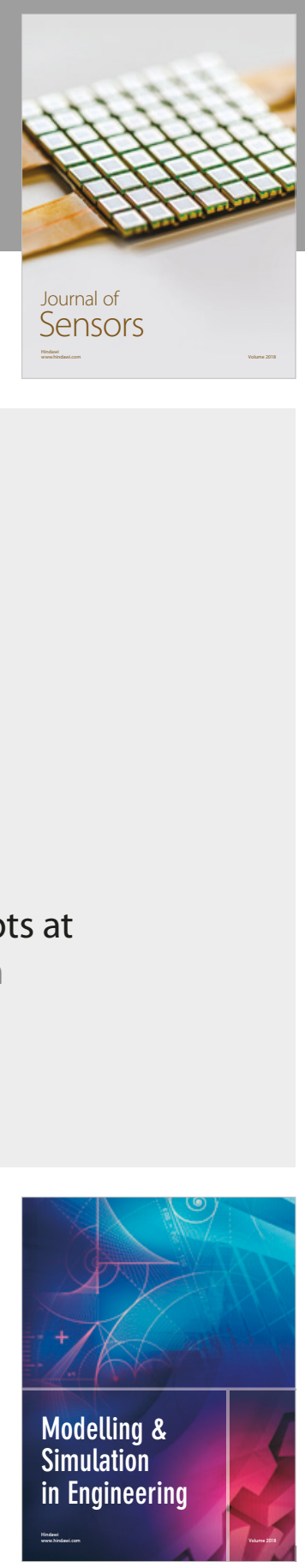

\section{Advances \\ Multimedia}
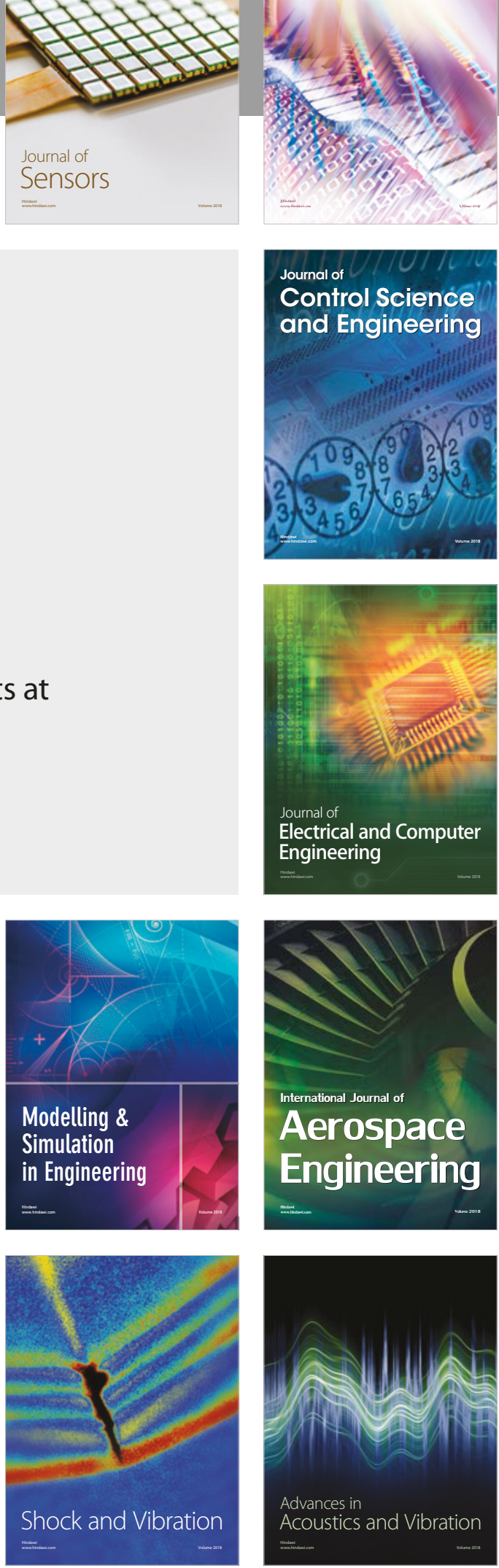